\title{
Two Node Communication Network Model with Weibull Inter arrival Times and Dynamic Bandwidth Allocation
}

\author{
G. Sita Ratnam \\ Department of CS \&SE, \\ Andhra University, \\ Visakhapatnam, \\ India
}

\author{
K. Srinivasa Rao \\ Department of Statistics, \\ Andhra University, \\ Visakhapatnam, \\ India
}

\author{
P. Srinivasa Rao \\ Department of CS \&SE, \\ Andhra University, \\ Visakhapatna \\ India
}

\begin{abstract}
In modeling the network traffic Poisson process is extensively used for packet and connection arrivals. Bit in internet traffic an arrival process is bursty due to selfsimilarity and time dependent nature. Rakesh Singhai et al(2007) have observed that Weibull distribution gives good fit for inter arrival times of packets in network traffic such as LAN, MAN, Wan and Web applications, which oneself similarity. In this paper for the first time we design a two node communication network with Weibull inter arrival times having Dynamic Band Width Allocation. Here, it is assumed that two transmitters are connected in tandem and packets arrive at the source are stored in buffers for forward transmission. The transmission strategy is dynamic band width allocation. The network performance measures such as average number of packets in each buffer, the mean delays in transmission, the throughput, the utilization of transmitters are derived explicitly. It is observed that the time dependent nature of mean arrival rate has significant influence on the performance measures. The dynamic band width allocation can reduce the congestion in buffers and mean delays in transmission. This model also include two node communication network model and exponential inter arrival times as a particular case.
\end{abstract}

\section{Keywords}

Weibull inter arrival times, Two node communication network, Performance evaluation of the network, Burstiness of buffers

\section{INTRODUCTION}

Communication Networks Models play a dominant role in design and analysis of networks. Recently, much work has been reported in literature regarding, modeling the communication networks / data voice transmission with various assumptions. In these models, it is customary to assume that packets or connection arrivals follow Poisson process because such process is simple and much theory is available with attractive properties (V.Frost and B. Melamed, (1994)). This is also supported by Abry et al ( 2002),Cappe et al (2002). Who stated that the Poisson like nature show that aggregate traffic smoother or less bursty. But the studies made by Jain and Routhier (1986), Gusella et al (1990), Fowler and Leland (1991), Danzig, Jamin, Caceres, Mitzel, and Estrin (1992) revealed that in the Local Area Network (LAN), Wide Area Network (WAN) and Metropolitan Area Network (MAN) traffic the packet arrival distribution is not exponential. Similarly Leland, et al (1994), have studied the LAN traffic can be better modeled by self similar processes which differ significantly from Poisson Process. Because in these networks the traffic burst appear over a wide range of time scales. Vern Paxson and Floyd (1995) have pointed that the Poisson Process, fails in modeling the WAN traffic, because of time dependent nature of the arrivals.

Recently, much emphasis is given for modeling the arrival process of the communication networks using, Nonhomogeneous Poisson Process with the assumption that the arrival rate is linearly dependent on time (M. V. Rama Sundari, K. Srinivasa Rao, P. Srinivasa Rao, P. Suresh Varma (2011), Trinatha Rao. P et al (2012), Suhasini, A. V. S. et al (2013a), Suhasini, A. V. S. et al (2013b)). In all these papers they assumed that the burstiness of the traffic is either linearly increasing or decreasing depending on time, but in LAN, WAN, and MAN the traffic structure is quite different from the, arrival rates being linearly dependent function of time. This is also supported by Rakesh Singhai et al (2007) who have, modeled the packet arrivals as Log Normal distributions, since, the associated network traffic inter arrivals are distributed with heavy tail distributions. Feldmann (2000) demonstrated that the Transport Control Protocol (TCP) connection arrival bursty and are characterized by heavy tail distributions such as Weibull distributions. Fisher et al (2001), has analyzed a singer server queueing model with Weibull inter arrivals using simulations studied. Araik Tamazian and Mikhail Bogachev (2015) have used Weibull distributions for inter arrival times in analyzing the performance of World Wide Web (WWW) servers. In these studies they consider that the server is single and the queues are independent.

But in LAN, WAN and MAN the output of one queue is an input to the other queue, and queues are not independent. No work has been reported in literature regarding Tandem communication network models with Weibull inter arrival times which model the LAN, WAN, MAN and other self similarity network traffic with time dependent bursts, more close to the reality. This motivated to develop and analyze a two node tandem communication network model with Weibull inter arrival times having dynamic band width allocation. The Weibull distribution includes exponential distribution and several other skewed distributions as particular cases. Using the differential calculus the explicit expressions for the network performance parameters such as mean number of packets in each buffer, mean delay in transmission, the throughput of the nodes, and the variability of the buffer content are derived. Through numerical analysis the performance of 
the communication network is evaluated with respect to the changes in input parameters. A comparative study of the proposed model with that of exponential inter arrival times is also carried.

\section{COMMUNICATION NETWORK MODEL AND TRANSIENT SOLUTION}

In this section a communication network model having two nodes in tandem is considered. The arrivals to the buffer connected at node 1 are assumed to follow a nonhomogeneous Duane process with mean arrival rate as power function of time $t$ and it is of the form $\chi(t)=a b t^{b-1}$. The transmission process from node one to node two follows a Poisson with a parameter $\mu_{1}$. After getting transmitted from node one the packets are forwarded to the second buffer for the transmission from second node and the transmission process of node two also follows a Poisson process with parameter $\mu_{2}$. There are no intermediate departure of packets after transmission from node- 1 . The transmission strategy in both the nodes are dynamic band width allocation. That is the transmission rate of each packet in each node is adjusted just before transmission depending on the content of the buffer connected to it. The packets are transmitted through the transmitters by the first in first out principles. The schematic diagram representing the communication network model is shown in "Figure1".

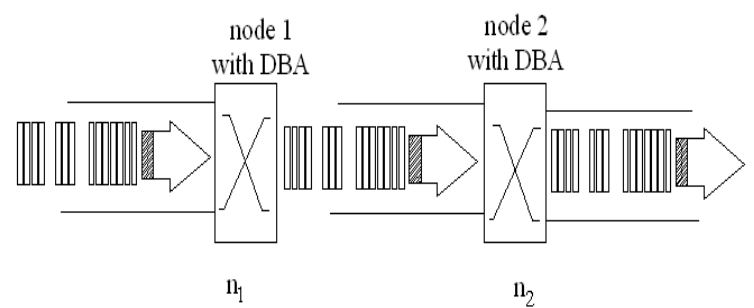

Figure 1: Schematic diagram of the CNM model with two stage arrival

Let $P n 1, n 2(t)$ be the probability that there are $n 1$ packets in the first buffer and $\mathrm{n} 2$ packets in the second buffer at time t. with this structure, the difference-differential equations of the communication network are:

$$
\begin{aligned}
& \frac{\partial p_{n_{1}, n_{2}}(t)}{\partial t}=-\left(\lambda(t)+n_{1} \mu_{1}+n_{2} \mu_{2}\right) p_{n_{1}, n_{2}}(t)+(\lambda(t)) p_{n_{1}-1, n_{2}}(t)+ \\
& \left(n_{1}+1\right) \mu_{1} p_{n_{1}+1, n_{2}-1}(t)+\left(n_{2}+1\right) \mu_{2} p_{n_{1}, n_{2}+1}(t) \\
& \frac{\partial p_{n_{1}, 0}(t)}{\partial t}=-\left(\lambda(t)+n_{1} \mu_{1}\right) p_{n_{1}, 0}(t)+(\lambda(t)) p_{n_{1}-1,0}(t)+\mu_{2} p_{n_{1}, 1}(t) \\
& \frac{\partial p_{0, n_{2}}(t)}{\partial t}=-\left(\lambda(t)+n_{2} \mu_{2}\right) p_{0, n_{2}}(t)+ \\
& \quad \mu_{1} p_{1, n_{2}-1}(t)+\left(n_{2}+1\right) \mu_{2} p_{0, n_{2}+1}(t) \\
& \frac{\partial p_{0,0}(t)}{\partial t}=-(\lambda(t)) p_{0,0}(t)+\mu_{2} p_{0,1}(t) \\
& \operatorname{LetP}\left(\mathrm{S}_{1}, S_{2}, t\right)=\sum_{n_{1}=0}^{\infty} \sum_{n_{2}=0}^{\infty} P_{n_{1}, n_{2}}(t) \cdot S_{1}^{n_{1}} \cdot S_{2}{ }^{n_{2}} \text { be }
\end{aligned}
$$

the joint probability generating function of $\mathrm{P}_{\mathrm{n} 1}, \mathrm{P}_{\mathrm{n} 2}(\mathrm{t})$ Multiplying the equation (1) with $\mathrm{S}_{1}{ }^{\mathrm{n} 1} \mathrm{~S}_{2}{ }^{\mathrm{n} 2}$ and summing overall $\mathrm{n}_{1}, \mathrm{n}_{2}$ we get

$$
\begin{aligned}
\frac{\partial P}{\partial t}=- & \sum_{n 1=0}^{\infty} \sum_{n 2=0}^{\infty}\left(\lambda(t)+n_{1} \mu_{1}+n_{2} \mu_{2}\right) P_{n 1, n 2}(t) \cdot S_{1}{ }^{n 1} \cdot S_{2}{ }^{n 2}+ \\
& \sum_{n 1=0}^{\infty} \sum_{n 2=0}^{\infty}(\lambda(t)) P_{n 1-1, n 2}(t) \cdot S_{1}{ }^{n 1} \cdot S_{2}{ }^{n 2}+ \\
& \sum_{n 1=0}^{\infty} \sum_{n 2=0}^{\infty}\left(\left(n_{1}+1\right) \mu_{1}\right) P_{n 1+1, n 2-1}(t) \cdot S_{1}{ }_{1} \cdot S_{2}{ }^{n 2}+ \\
& \sum_{n 1=0}^{\infty} \sum_{n 2=0}^{\infty}\left(\left(n_{2}+1\right) \mu_{2}\right) P_{n 1, n 2+1}(t) \cdot S_{1}{ }^{n 1} \cdot S_{2}{ }^{n 2}
\end{aligned}
$$

After simplifying, we get

$$
\frac{\partial P}{\partial t}=\mu_{1} \frac{\partial P}{\partial S_{1}}\left(S_{2}-S_{1}\right)+\mu_{2} \frac{\partial P}{\partial S 2}\left(1-S_{2}\right)-\lambda(t) P\left(1-S_{1}\right)
$$

Solving the equation (6) by Lagrangin's method the auxulary equations are

$$
\frac{d t}{1}=\frac{d s_{1}}{-\mu_{1}\left(s_{2}-s_{1}\right)}=\frac{d s_{2}}{-\mu_{2}\left(1-s_{2}\right)}=\frac{d p}{\lambda(t) p\left(s_{1}-1\right)}
$$

To solve the equation (7) the functional form of $\lambda(t)$ is required.

The mean arrival rate of packets is

$$
\lambda(t)=a b t^{b-1} \text { where, } \lambda>0, \mathrm{a}, \mathrm{b} \text { are constants. }
$$

Solving the first and third terms in equation (7), we get

$$
A=\left(s_{2}-1\right) e^{-\mu_{2} t}
$$

Solving first and second terms in equation (7) we get

$$
B=\left(s_{1}-1\right) e^{\mu_{1} t}+\frac{\mu_{1\left(S_{2}-1\right)}}{\mu_{2}-\mu_{1}} e^{-\mu_{1} t}
$$

Solving the first and fourth terms in equation (7) we get,

$$
C=P . \exp \left[a \cdot b\left[\begin{array}{c}
\left(\left(s_{1}-1\right) e^{-\mu_{1} \cdot t} \int_{0}^{t} e^{-\mu_{1} \cdot t} \cdot v^{b-1} \cdot d v\right) \\
+\left(\frac{\mu_{1}\left(s_{2}-1\right)}{\left(\mu_{2}-\mu_{1}\right)} e^{-\mu_{1} \cdot t} \int_{0}^{t} e^{\mu_{1} \cdot t} \cdot v^{b-1} \cdot d v\right)- \\
\left(\frac{\mu_{1}}{\left(\mu_{2}-\mu_{1}\right)}\left(s_{2}-1\right) e^{-\mu_{2} \cdot t} \int_{0}^{t} e^{\mu_{2} \cdot t} \cdot v^{b-1} \cdot d v\right)
\end{array}\right]\right.
$$

where A,B,C are arbitrary constants using the initial conditions $\mathrm{P}_{0,0} \quad(0)=1, \mathrm{P}_{0,0} \quad(\mathrm{t})=1$, for all $\mathrm{t}>\mathrm{o}$.The general solution of the equation (6) gives the Probability generating function of the number of packets in the first buffer and the number of packets in the second buffer at time $\mathrm{t}$ as $\mathrm{P}\left(\mathrm{S}_{1}, \mathrm{~S}_{2}, \mathrm{t}\right)$ 


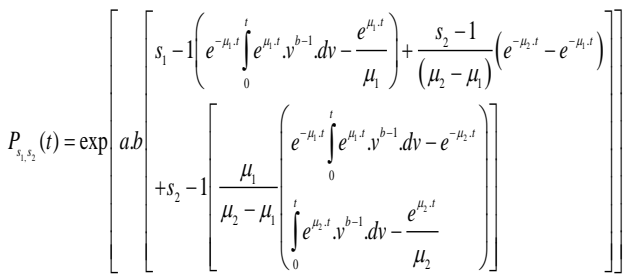

\section{PERFORMANCE MEASURES OF THE NETWORK}

In this section, we derive and analyze the performance measures of the communication network under transient conditions. .Expanding $\mathrm{P}\left(\mathrm{s}_{1}, \mathrm{~s}_{2}, \mathrm{t}\right)$ given in equation (11) and collecting the constant terms, we get the probability that the network is empty as

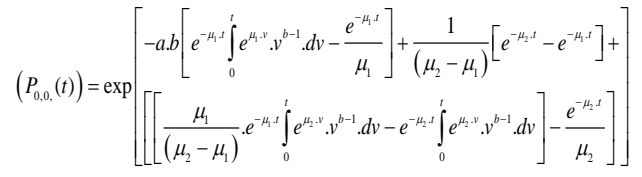

Taking $s_{2}=1$, the probability generating function of the first buffer size distribution is

$$
P_{0,0,}(t)=\exp \left[a \cdot b\left[\left(s_{1}-1\right) e^{-\mu_{1} \cdot t} \int_{0}^{t} e^{\mu_{1} \cdot v} \cdot v^{b-1} \cdot d v-\frac{e^{-\mu_{1} \cdot t}}{\mu_{1}}\right]\right]
$$

Expanding $\mathrm{P}\left(\mathrm{s}_{1}, \mathrm{t}\right)$ and collecting the constant terms we get the probability that the first buffer is empty as

$$
P_{0 .}(t)=\exp \left[-\left[a \cdot b\left(e^{-\mu_{1} \cdot t} \int_{0}^{t} e^{\mu_{1} \cdot v} \cdot v^{b-1} \cdot d v-\frac{e^{-\mu_{1} \cdot t}}{\mu_{1}}\right)\right]\right]
$$

The mean numbers of packets in the first buffer is

$$
L_{1}=E\left[N_{1}\right]=\sum_{n_{1}=0}^{\infty} n_{1} P_{n_{1}}(t)=a \cdot b\left[e^{-\mu_{1} \cdot t} \int_{0}^{t} e^{\mu_{1} \cdot v} \cdot v^{b-1} \cdot d v-\frac{e^{-\mu_{1} . t}}{\mu_{1}}\right]
$$

The utilization of the first buffer is

$U_{1}=1-P_{0}(t)=\left[1-\exp \left[-a \cdot b\left(e^{-\mu_{1} \cdot t} \int_{0}^{t} e^{\mu_{1} \cdot v} \cdot v^{b-1} \cdot d v-\frac{e^{-\mu_{1} . t}}{\mu_{1}}\right)\right]\right]$

for computing the variability of the number of packets in first buffer, we compute

$$
E\left(N_{1}^{2}-N_{1}\right)=a \cdot b\left[e^{-\mu_{1} \cdot t} \int_{0}^{t} e^{\mu_{1} \cdot v} \cdot v^{b-1} \cdot d v-\frac{e^{-\mu_{1} \cdot t}}{\mu_{1}}\right]^{2}
$$

Therefore, the variance of the number of packets in the first buffer is

$\operatorname{Var}\left(N_{1}\right)=a \cdot b\left[e^{-\mu_{1} \cdot t} \int_{0}^{t} e^{\mu_{1} \cdot v} \cdot v^{b-1} \cdot d v-\frac{e^{-\mu_{1} \cdot t}}{\mu_{1}}\right]$

The throughput of the first transmitter is

$$
\mu_{1}\left(1-P_{0 .}(t)\right)=\mu_{1}\left[1-\exp \left[-a . b\left(e^{-\mu_{1}, t} \int_{0}^{t} e^{\mu_{1}, v} \cdot v^{b-1} \cdot d v-\frac{e^{-\mu_{1}, t}}{\mu_{1}}\right)\right]\right]
$$

The mean delay in the first buffer size is

$$
\begin{aligned}
& W_{1}=\frac{L_{1}}{\boldsymbol{T h} p_{1}}=\frac{E\left[N_{1}\right]}{\boldsymbol{T h p _ { 1 }}} \\
& =\frac{a \cdot b\left[e^{-\mu_{1} \cdot t} \int_{0}^{t} e^{\mu_{1} \cdot v} \cdot v^{b-1} \cdot d v-\frac{e^{-\mu_{1} \cdot t}}{\mu_{1}}\right]}{\left[1-\exp \left[-\left[a \cdot b\left(e^{-\mu_{1} \cdot t} \int_{0}^{t} e^{\mu_{1} \cdot v} \cdot v^{b-1} \cdot d v-\frac{e^{-\mu_{1} \cdot t}}{\mu_{1}}\right)\right]\right]\right]}
\end{aligned}
$$

Similarly by substituting $s 1=1$, we get the probability generating function of the second buffer size distribution as

$$
P_{s_{1}, s_{2}}(t)=\exp \left[a \cdot b\left[\begin{array}{l}
\frac{s_{2}-1}{\left(\mu_{2}-\mu_{1}\right)}\left(e^{-\mu_{2} \cdot t}-e^{-\mu_{1} \cdot t}\right)+ \\
s_{2}-1\left[\begin{array}{l}
\frac{\mu_{1}}{\mu_{2}-\mu_{1}} \cdot e^{-\mu_{1} \cdot t} \int_{0}^{t} e^{\mu_{1} \cdot t} \cdot v^{b-1} d v \\
-e^{-\mu_{2} \cdot t} \int_{0}^{t} e^{\mu_{2} \cdot t} \cdot v^{b-1} \cdot d v-\frac{e^{\mu_{2} \cdot t}}{\mu_{2}}
\end{array}\right]
\end{array}\right]\right]
$$

Expanding $\mathrm{P}(\mathrm{s} 2, \mathrm{t})$ and collecting the constant terms, we get the probability that the second buffer is empty as

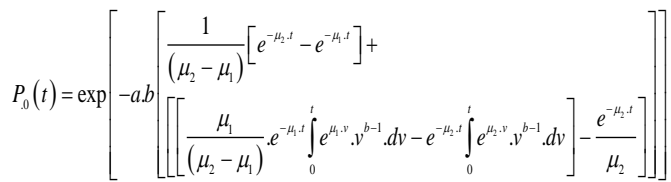

The mean number of packets in the second buffer is

$L_{2}=a \cdot b\left[\begin{array}{l}\frac{1}{\left(\mu_{2}-\mu_{1}\right)}\left[e^{-\mu_{2} \cdot t}-e^{-\mu_{1} \cdot t}\right]+\frac{\mu_{1}}{\left(\mu_{2}-\mu_{1}\right)} . \\ e^{-\mu_{1} \cdot t} \int_{0}^{t} e^{\mu_{1} \cdot v} \cdot v^{b-1} \cdot d v-e^{-\mu_{2} \cdot t} \int_{0}^{t} e^{\mu_{2} \cdot v} \cdot v^{b-1} \cdot d v-\frac{e^{-\mu_{2} \cdot t}}{\mu_{2}}\end{array}\right]$

The utilization of the second buffer is

$$
U t p_{2}=\left[1-\exp \left[-a \cdot b\left(\begin{array}{l}
\frac{1}{\left(\mu_{2}-\mu_{1}\right)}\left[e^{-\mu_{2}, t}-e^{-\mu_{1}, t}\right]+\frac{\mu_{1}}{\left(\mu_{2}-\mu_{1}\right)} . \\
e^{-\mu_{1} \cdot t} \int_{0}^{t} e^{\mu_{2}, v} \cdot v^{b-1} \cdot d v-e^{-\mu_{2}, t} \int_{0}^{t} e^{\mu_{2}, v} \cdot v^{b-1} \cdot d v-\frac{e^{-\mu_{2}, t}}{\mu_{2}}
\end{array}\right]\right]\right]
$$

For computing the variability of the number of packets in second buffer, we compute

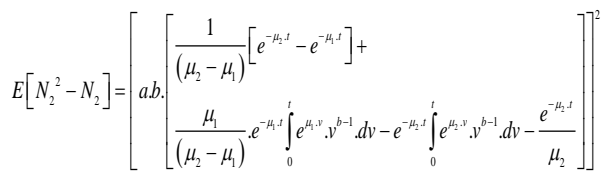

The variance of the number of packets in the second buffer

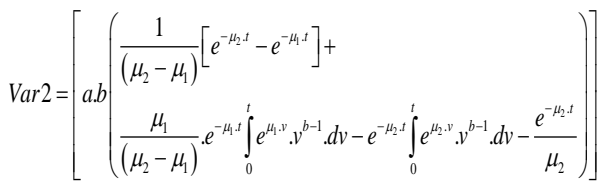

The throughput of the second transmitter is 


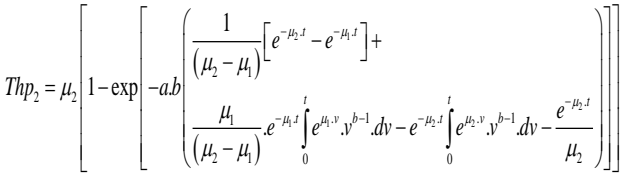

The mean delay in the second buffer is

$$
\begin{aligned}
& W_{2}=\frac{L_{1}}{T h p}=\frac{E\left(N_{2}\right)}{T h p}
\end{aligned}
$$

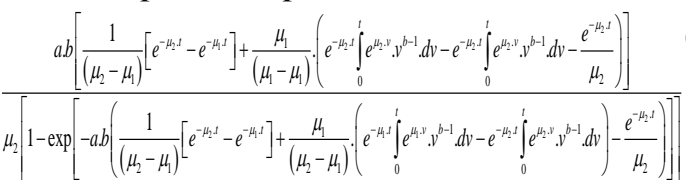

From the equations (15) and (23) we obtain

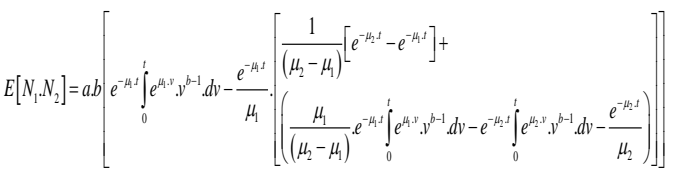

The mean number of packets in the network at time $t$ is $\mathrm{L}$ (t)

$\mathrm{L}(\mathrm{t})=\mathrm{E}[\mathrm{N} 1]+\mathrm{E}[\mathrm{N} 2]$. This implies

$$
\begin{aligned}
& L(t)=a . b\left[e^{-\mu_{1} \cdot t} \int_{0}^{t} e^{\mu_{1} \cdot v} \cdot v^{b-1} \cdot d v-\frac{e^{-\mu_{1}, t}}{\mu_{1}}\right]+ \\
& a . b\left[\begin{array}{l}
\frac{1}{\left(\mu_{2}-\mu_{1}\right)}\left[e^{-\mu_{2} \cdot t}-e^{-\mu_{1} \cdot t}\right]+ \\
\frac{\mu_{1}}{\left(\mu_{2}-\mu_{1}\right)} \cdot e^{-\mu_{1} \cdot t} \int_{0}^{t} e^{\mu_{1} \cdot v} \cdot v^{b-1} \cdot d v- \\
\mu_{0}^{t} e^{\mu_{2} \cdot v} \cdot v^{b-1} \cdot d v-\frac{e^{-\mu_{2} \cdot t}}{\mu_{2}}
\end{array}\right]
\end{aligned}
$$

The variability of the number of packets in the network is

$$
\begin{aligned}
& \operatorname{Var}[N]=\operatorname{var}\left[N_{1}\right]+\operatorname{var}\left[N_{2}\right]+2 \operatorname{Cov}\left[N_{1,} N_{2}\right] \\
& =a \cdot b\left[e^{-\mu_{1} \cdot t} \int_{0}^{t} e^{\mu_{1} \cdot v} \cdot v^{b-1} \cdot d v-\frac{e^{-\mu 1 . t}}{\mu_{1}}\right]+\frac{1}{\left(\mu_{2}-\mu_{1}\right)}\left[e^{-\mu_{2} \cdot t}-e^{-\mu_{1} \cdot t}\right] \\
& +\left[\left[\frac{\mu_{1}}{\left(\mu_{2}-\mu_{1}\right)} \cdot e^{-\mu_{1} \cdot t} \int_{0}^{t} e^{\mu_{2} \cdot v} \cdot v^{b-1} \cdot d v-e^{-\mu_{2} \cdot t} \int_{0}^{t} e^{\mu_{2} \cdot v} \cdot v^{b-1} \cdot d v\right]-\frac{e^{-\mu_{2} \cdot t}}{\mu_{2}}\right]
\end{aligned}
$$

\section{PERFORMANCE EVALUATION OF THE NETWORK}

In this section, the performance of the proposed communication network is discussed through numerical illustration. Different values of the parameters are considered for bandwidth allocation and arrival of packets. After interacting with the technical staff at internet providing station, it is considered the packet arrival parameter (a) varies from $8 \times 10^{4}$ packets/sec to $11 \times 10^{4}$ packets/sec, and b varies from 1.25 to 2 with an average packet size of 111 bytes. After transmitting from node 1 , the forward transmission rate $\mu_{1}$ varies from $11 \times 10^{4}$ packets $/ \mathrm{sec}$ to $14 \times 10^{4}$ packets $/ \mathrm{sec}$. The rate of transmission rate $\mu_{2}$,varies from $60 \times 10^{4}$ packets $/ \mathrm{sec}$ to $90 \times 10^{4}$ packets/sec. In all these nodes, dynamic bandwidth allocation strategy is considered i.e., the transmission rate of each packet depends on the number of packets in the buffer connected to it at that instant. Since performance characteristics of communication network are highly sensitive with respect to time, the transient behavior of the model is studied through computing the performance measures. The probability of the network emptiness and buffers emptiness, utilization of transmitters are computed for different values of t,a,b $\mu_{1}, \mu_{2}$, and presented in Table1 and Figure 2.

It is observed that the probability of the emptiness in the entire communication network and in the two buffers is highly sensitive with respect to changes in time. As time (t) varies from 0.5 seconds to 5 seconds, the probability of the emptiness in the network reduces from 0.6079 to 0.00054 when other parameters are fixed at $(9,2,10,50)$ for $\left(\mathrm{a}, \mathrm{b}, \mu_{1}, \mu_{2}\right)$. Similarly the probability for the emptiness in the 2 buffers varies from 0.6973 to $0.0011,0.8719$ to 0.5082 for node 1 and node 2 respectively. The decrease in the node 1 is more rapid when compared to node 2 .

Table 1: Values of network and buffer emptiness probability and utilization of the communication network with dynamic bandwidth allocation.

\begin{tabular}{|l|l|l|l|l|l|l|l|l|l|}
\hline $\mathbf{t}$ & $\mathbf{a}$ & $\mathbf{b}$ & $\boldsymbol{\mu}_{\mathbf{1}}$ & $\boldsymbol{\mu}_{\mathbf{2}}$ & $\mathbf{P}_{\mathbf{0 , 0}}(\mathbf{t})$ & $\mathbf{P}_{\mathbf{0 ,}}(\mathbf{t})$ & $\mathbf{P}_{., \mathbf{0}}(\mathbf{t})$ & $\mathbf{U}_{\mathbf{1}}$ & $\mathrm{U}_{2}$ \\
\hline $\mathbf{0 . 5}$ & 9 & 2 & 10 & 50 & 0.6079 & 0.6973 & 0.872 & 0.3027 & 0.1281 \\
\hline $\mathbf{1}$ & 9 & 2 & 10 & 50 & 0.1442 & 0.1979 & 0.729 & 0.8021 & 0.2715 \\
\hline $\mathbf{1 . 5}$ & 9 & 2 & 10 & 50 & 0.0139 & 0.0228 & 0.609 & 0.9772 & 0.3915 \\
\hline $\mathbf{2}$ & 9 & 2 & 10 & 50 & 0.0005 & 0.0011 & 0.508 & 0.9989 & 0.4918 \\
\hline 1.5 & $\mathbf{8}$ & 2 & 10 & 50 & 0.0223 & 0.0347 & 0.643 & 0.9653 & 0.357 \\
\hline 1.5 & $\mathbf{9}$ & 2 & 10 & 50 & 0.0139 & 0.0228 & 0.609 & 0.9772 & 0.3915 \\
\hline 1.5 & $\mathbf{1 0}$ & 2 & 10 & 50 & 0.0086 & 0.015 & 0.576 & 0.985 & 0.4242 \\
\hline 1.5 & $\mathbf{1 1}$ & 2 & 10 & 50 & 0.0054 & 0.0098 & 0.545 & 0.9901 & 0.4551 \\
\hline 1.5 & 9 & $\mathbf{1 . 2 5}$ & 10 & 50 & 0.1251 & 0.1597 & 0.784 & 0.8403 & 0.2163 \\
\hline 1.5 & 9 & $\mathbf{1 . 5}$ & 10 & 50 & 0.0665 & 0.0912 & 0.728 & 0.9088 & 0.2716 \\
\hline 1.5 & 9 & $\mathbf{1 . 7 5}$ & 10 & 50 & 0.0834 & 0.1075 & 0.776 & 0.8925 & 0.3302 \\
\hline 1.5 & 9 & $\mathbf{2}$ & 10 & 50 & 0.0139 & 0.0228 & 0.609 & 0.9772 & 0.3915 \\
\hline 1.5 & 9 & 2 & $\mathbf{1 1}$ & 50 & 0.0191 & 0.0315 & 0.607 & 0.9685 & 0.3935 \\
\hline 1.5 & 9 & 2 & $\mathbf{1 2}$ & 50 & 0.025 & 0.0413 & 0.605 & 0.9587 & 0.3952 \\
\hline 1.5 & 9 & 2 & $\mathbf{1 3}$ & 50 & 0.0314 & 0.052 & 0.603 & 0.948 & 0.3966 \\
\hline 1.5 & 9 & 2 & $\mathbf{1 4}$ & 50 & 0.0383 & 0.0636 & 0.602 & 0.9364 & 0.3978 \\
\hline 1.5 & 9 & 2 & 12 & $\mathbf{6 0}$ & 0.0271 & 0.0413 & 0.657 & 0.9587 & 0.343 \\
\hline 1.5 & 9 & 2 & 12 & $\mathbf{7 0}$ & 0.0288 & 0.0413 & 0.697 & 0.9587 & 0.3082 \\
\hline 1.5 & 9 & 2 & 12 & $\mathbf{8 0}$ & 0.0301 & 0.0413 & 0.729 & 0.9587 & 0.2709 \\
\hline 1.5 & 9 & 2 & 12 & $\mathbf{9 0}$ & 0.0312 & 0.0413 & 0.755 & 0.9587 & 0.2451 \\
\hline & & & & & & & & \\
\hline
\end{tabular}

*=Seconds, \$=Multiples of 10,000 packets/sec

The influence of arrival rate parameters on the system emptiness is also studied. As the arrival parameter (a) varies from $8 \times 10^{4}$ packets $/ \mathrm{sec}$ to $11 \times 10^{4}$ packets $/ \mathrm{sec}$, the probability of emptiness of the network decreases from 0.0223 to 0.00536 when other parameters are fixed at $(1.5,2,10,50)$ for $\left(t, b, \mu_{1}, \mu_{2}\right)$.The same phenomenon is observed with respect to both nodes. This decrease is more than in first node and moderate in the second node. When the arrival parameter (b) varies from 1.25 to 2 , the probability of emptiness of the network decreases from 0.1251 to 0.0139 when the other parameters remain fixed. The same phenomenon is observed for both the nodes. The decrease is more fast at node 1 and moderate in the next node. When the transmission rate $\mu_{1}$ of node 1 varies from 
$11 \times 10^{4}$ packets/sec to $14 \times 10^{4}$ packets/sec the probability of emptiness of the network increases from 0.0191 to 0.0383 when the other parameter remain fixed. Similarly the transmission rate $\mu_{2}$ of node 2 varies from $60 \times 10^{4}$ packets $/ \mathrm{sec}$ to $90 \times 10^{4}$ packets/sec. The probability of emptiness of the network increases from 0.0271 to 0.0312 when the other parameters remain fixed. The same phenomenon is observed with respect to the emptiness of the two buffers. It is also observed that the as the arrival rate parameter (a) increases the utilization of transmitters at both nodes are increasing for fixed values of the other parameters.

As the transmission rate $\mu_{1}$ increases, the utilization of the first node decreases when the other parameters remain fixed. Similarly as the transmission rate $\mu_{2}$ increases the utilization of the second node decreases when the other parameters remain fixed.
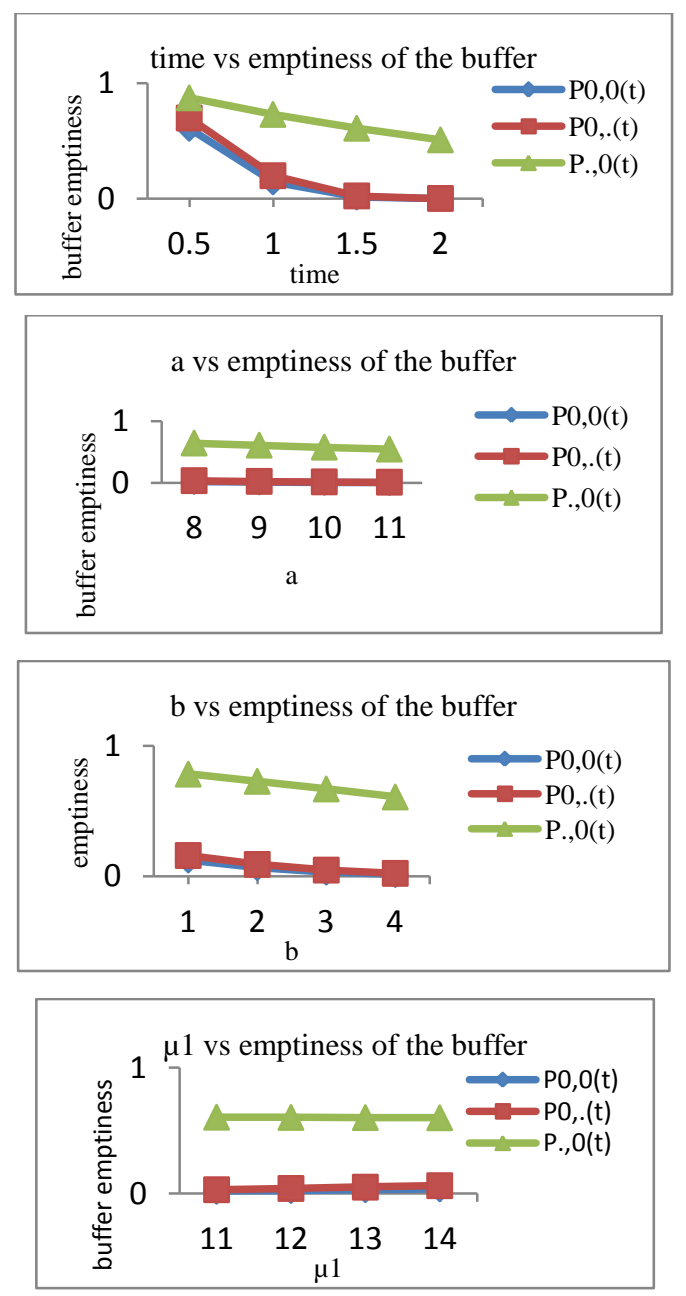
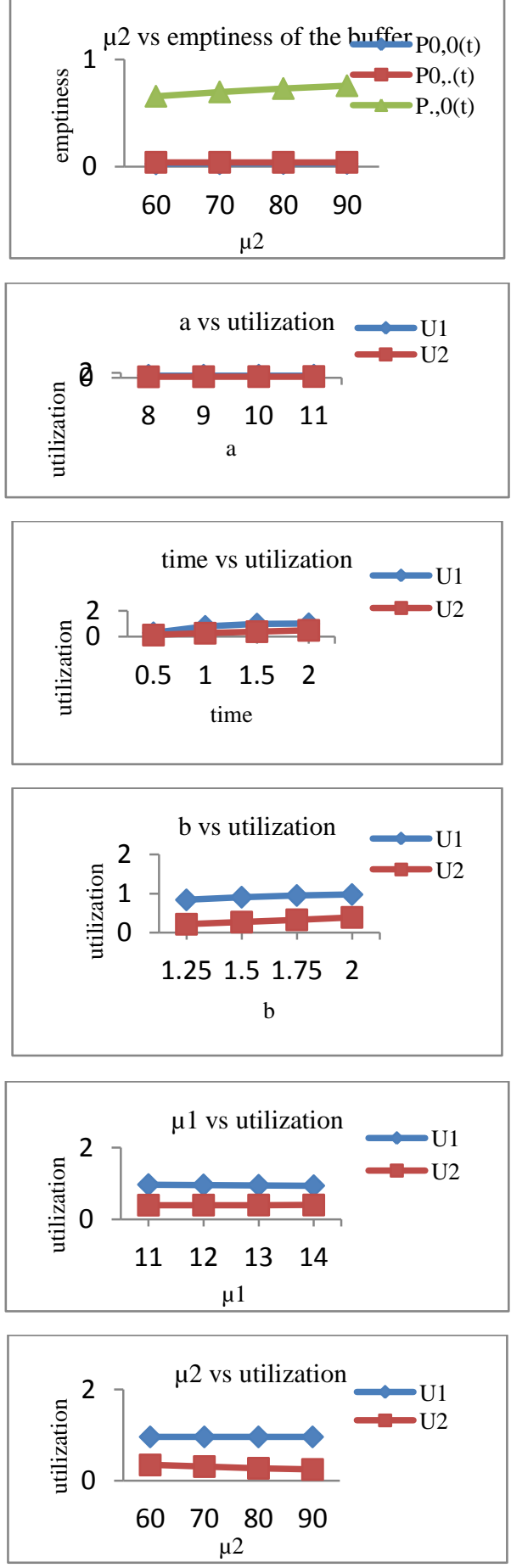

Figure 2: The relationship between network emptiness, utilization and various parameters

The mean number of packets in the buffers and in the network mean delays in transmission of both nodes are computed for different values are t,a,b, $\mu_{1}, \mu_{2}$ and presented at Table 2 and "figure 3".It is observed that at 0.5 seconds, the first buffer contains an average of 3606 packets, after 1 second it is rapidly increased to an average of 16200 packets. After of 1.5 seconds, the first buffer contains an average of 37800 packets and thereafter there is steady increase in the content of buffer for fixed values of other parameters $(9,2,10,50)$ for $(a, b$, $\left.\mu_{1}, \mu_{2}\right)$. It is also observed that as time $(\mathrm{t})$ varies from 0.5 seconds to 2 seconds, the average content of the second buffer and in the network are increasing from 1371 packets to 6768 packets and from 4977 packets to 75168 packets respectively when other parameters remain fixed.When the arrival rate parameter (a) varies from $8 \times 10^{4}$ packets $/ \mathrm{sec}$ to $11 \times 10^{4}$ packets $/ \mathrm{sec}$, the average content of the first buffer, second buffer and in the network are increasing from 33600 packets to 46200 packets, 4416 packets to 6072 packets, 38016 packets to 52272 packets respectively when other parameters remain fixed. The arrival rate parameter (b) varies from 1.25 to 2 
the average number of packets in the first buffer, second buffer and in the network are increasing from 18346 packets to 37800 packets, 2437 packets to 4968 packets and 20783 packets to 42768 packets respectively when other parameters remain fixed.When the transmission rate $\mu_{1}$ varies from $11 \times 10^{4}$ packets/sec to $14 \times 10^{4}$ packets/sec, the average content of the first buffer and in the network are decreasing from 34587 packets to 27551 packets from 39588 packets to 32622 packets and the average content in the second buffer increasing from 5001 packets to 5071 packets respectively when other parameters remain fixed. Similarly the transmission rate $\mu_{2}$ varies from $60 \times 10^{4}$ packets/sec to $90 \times 10^{4}$ packets/sec, the average content of the first buffer remains fixed at 31875 packets and the average content of second buffer and in the network are decreasing from 4200 packets to 2811 packets and 36075 packets to 34686 packets respectively when other parameters are fixed. It is observed that as the time ( $\mathrm{t}$ ) and the arrival rate parameter (a) are increasing, the mean delay in buffers is increasing from fixed values of the other parameters. When the transmission rate $\mu_{1}$ increases, the mean delay in the first buffer decreases when the other parameters remains fixed. Similarly, the transmission rate $\mu_{2}$ increases the mean delay in the second buffer decreases when the other parameter remains fixed.

Table 2: Values of mean number of packets and mean delay of the communication networks with dynamic band width allocation and Bulk Arrivals

\begin{tabular}{|c|c|c|c|c|c|c|c|c|l|l|l|l|}
\hline $\mathbf{t}^{*}$ & $\mathbf{a} \$$ & $\mathbf{B}$ & $\boldsymbol{\mu}_{1} \mathbf{\$}$ & $\boldsymbol{\mu}_{2} \mathbf{\$}$ & $\mathrm{P}_{0,0}(\mathrm{t})$ & $\mathrm{P}_{0, .}(\mathrm{t})$ & $\mathbf{P}_{., 0}(\mathrm{t})$ & $\mathbf{L}_{\mathbf{1}}$ & $\mathbf{L}_{\mathbf{2}}$ & $\mathbf{L N}$ & $\mathbf{W}_{\mathbf{1}}$ & $\mathbf{W}_{\mathbf{2}}$ \\
\hline 0.5 & 9 & 2 & 10 & 50 & 0.6079 & 0.6973 & 0.8719 & 0.3606 & 0.1371 & 0.4977 & 0.1191 & 0.0214 \\
\hline 1 & 9 & 2 & 10 & 50 & 0.1442 & 0.1979 & 0.7285 & 1.6200 & 0.3168 & 1.9368 & 0.2020 & 0.0233 \\
\hline 1.5 & 9 & 2 & 10 & 50 & 0.0139 & 0.0228 & 0.6085 & 3.7800 & 0.4968 & 4.2768 & 0.3868 & 0.0254 \\
\hline 2 & 9 & 2 & 10 & 50 & 0.00054 & 0.0011 & 0.5082 & 6.8400 & 0.6768 & 7.5168 & 0.6847 & 0.0275 \\
\hline 1.5 & 8 & 2 & 10 & 50 & 0.0223 & 0.0347 & 0.643 & 3.3600 & 0.4416 & 3.8016 & 0.3481 & 0.0247 \\
\hline 1.5 & 9 & 2 & 10 & 50 & 0.0139 & 0.0228 & 0.6085 & 3.7800 & 0.4968 & 4.2768 & 0.3868 & 0.0254 \\
\hline 1.5 & 10 & 2 & 10 & 50 & 0.00863 & 0.015 & 0.5758 & 4.2000 & 0.5520 & 4.7520 & 0.4264 & 0.0260 \\
\hline 1.5 & 11 & 2 & 10 & 50 & 0.00536 & 0.0098 & 0.5449 & 4.6200 & 0.6072 & 5.2272 & 0.4666 & 0.0267 \\
\hline 1.5 & 9 & 1.25 & 10 & 50 & 0.1251 & 0.1597 & 0.7837 & 1.8346 & 0.2437 & 2.0783 & 0.2183 & 0.0225 \\
\hline 1.5 & 9 & 1.5 & 10 & 50 & 0.0665 & 0.0912 & 0.7284 & 2.3943 & 0.3169 & 2.7112 & 0.2635 & 0.0233 \\
\hline 1.5 & 9 & 1.75 & 10 & 50 & 0.0834 & 0.1075 & 0.7756 & 2.2305 & 0.2541 & 2.4846 & 0.2499 & 0.0226 \\
\hline 1.5 & 9 & 2 & 10 & 50 & 0.0139 & 0.0228 & 0.6085 & 3.7800 & 0.4968 & 4.2768 & 0.3868 & 0.2540 \\
\hline 1.5 & 9 & 2 & 11 & 50 & 0.0191 & 0.0315 & 0.6065 & 3.4587 & 0.5001 & 3.9588 & 0.3246 & 0.0254 \\
\hline 1.5 & 9 & 2 & 12 & 50 & 0.0250 & 0.0413 & 0.6048 & 3.1875 & 0.5028 & 3.6903 & 0.2771 & 0.6254 \\
\hline 1.5 & 9 & 2 & 13 & 50 & 0.0314 & 0.0520 & 0.6034 & 2.9556 & 0.5051 & 3.4607 & 0.2398 & 0.0255 \\
\hline 1.5 & 9 & 2 & 14 & 50 & 0.0383 & 0.0636 & 0.6022 & 2.7551 & 0.5071 & 3.2622 & 0.2102 & 0.0255 \\
\hline 1.5 & 9 & 2 & 12 & 60 & 0.0271 & 0.0413 & 0.657 & 3.1875 & 0.4200 & 3.6075 & 0.2771 & 0.0204 \\
\hline 1.5 & 9 & 2 & 12 & 70 & 0.0288 & 0.0413 & 0.6972 & 3.1875 & 0.3606 & 3.5481 & 0.2771 & 0.0170 \\
\hline 1.5 & 9 & 2 & 12 & 80 & 0.0301 & 0.0413 & 0.7291 & 3.1875 & 0.3159 & 3.5034 & 0.2771 & 0.0146 \\
\hline 1.5 & 9 & 2 & 12 & 90 & 0.0312 & 0.0413 & 0.7549 & 3.1875 & 0.2811 & 3.4686 & 0.2771 & 0.0127 \\
\hline
\end{tabular}

*=Seconds, \$=Multiples of 10,000 packets/sec,
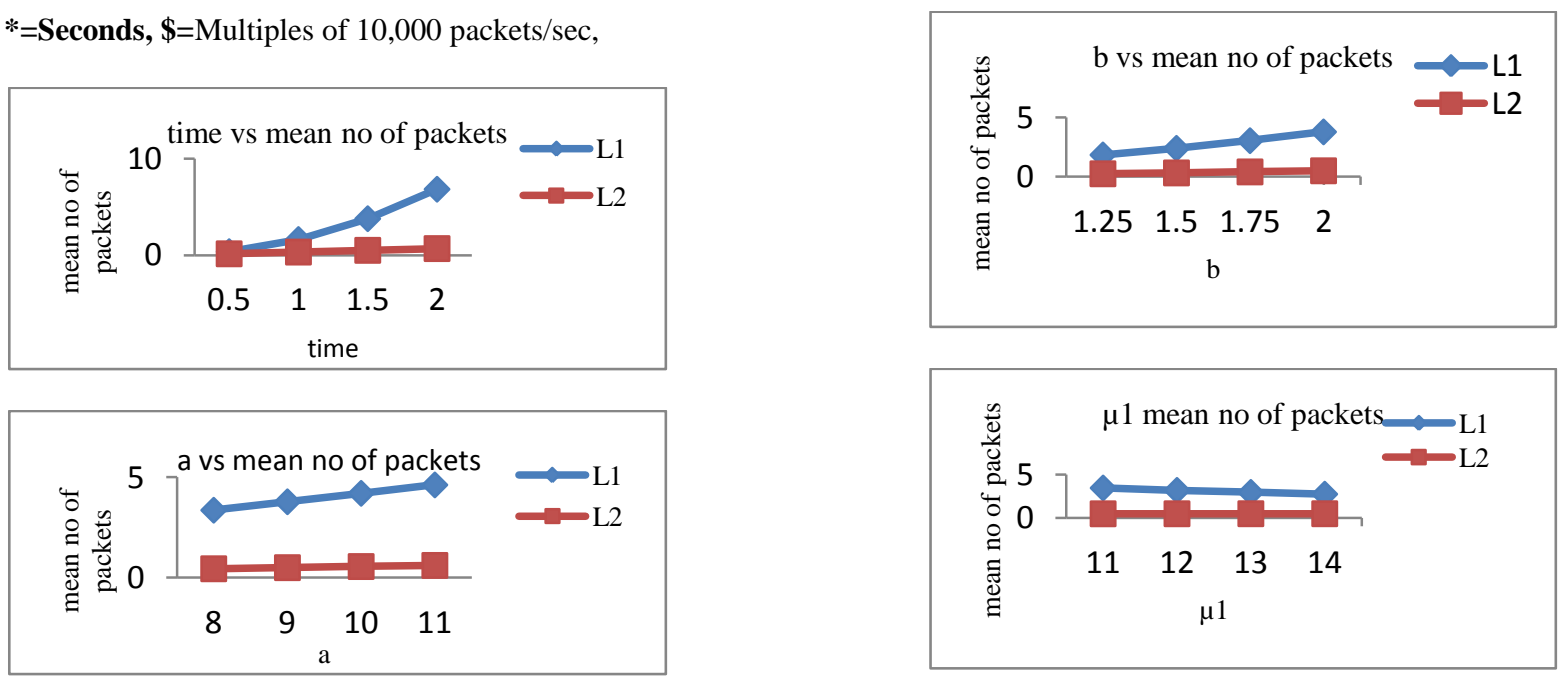

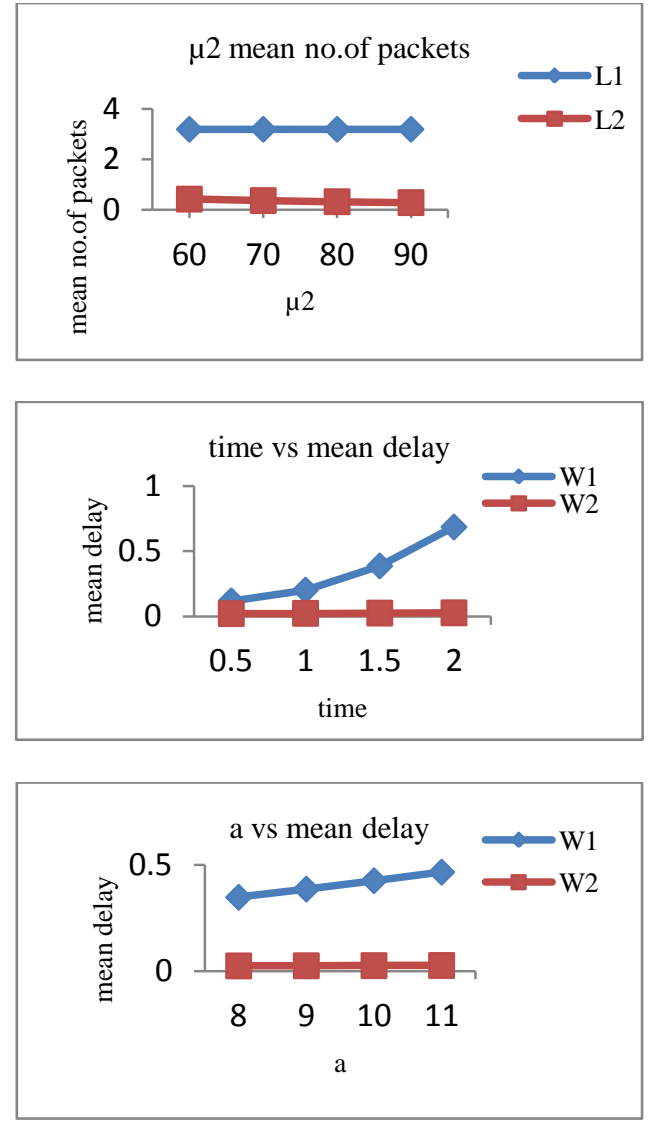
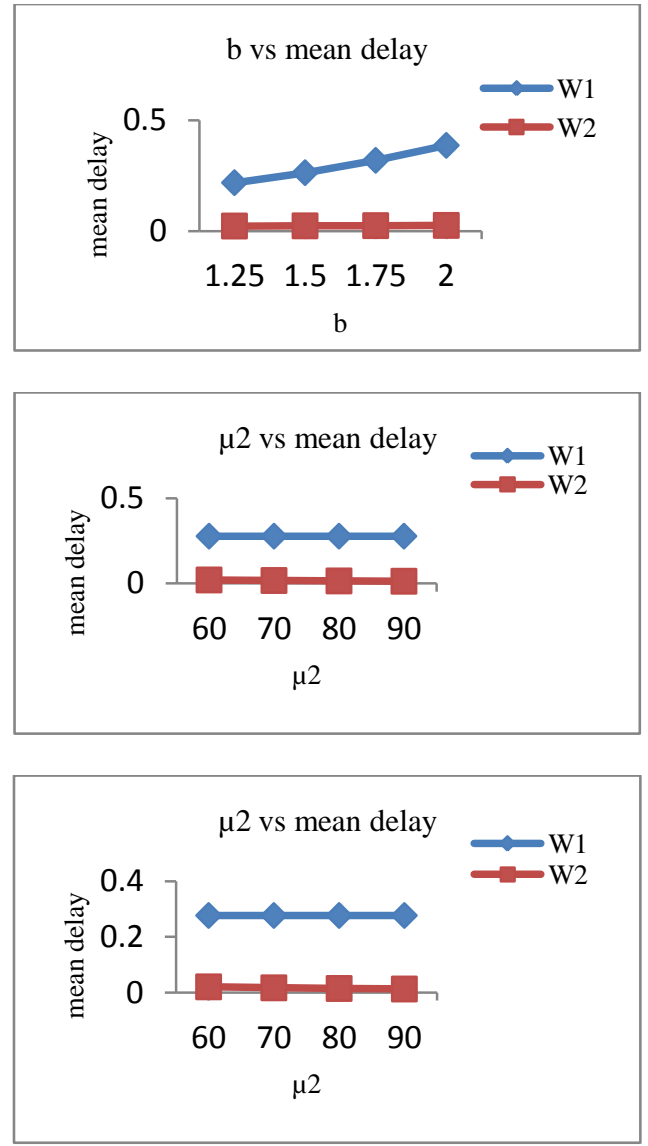

Figure 3: The relationship between mean number of packets, mean delay and various parameters

The variance of the number of packets in each buffer and throughput of each node are computed for different values of t,a,b, $\mu_{1}, \mu_{2}$, and presented in "Table 3 " and "figure 4".

Table 3: Effect of various parameters on Throughput and variance under transient state for communication network model with two stage arrivals

\begin{tabular}{|c|c|c|c|c|c|c|c|c|c|c|c|}
\hline $\mathbf{t} *$ & $\mathbf{a} \$$ & $\mathbf{b}$ & $\mu_{1} \mathbf{\$}$ & $\mu_{2} \$$ & $\mathbf{P}_{0,0}(\mathrm{t})$ & $\mathbf{P}_{0, .}(\mathrm{t})$ & $\mathbf{P}_{., 0}(\mathrm{t})$ & Thp1 & Thp2 & Var(N1) & Var(N2) \\
\hline 0.5 & 9 & 2 & 10 & 50 & 0.60790 & 0.6973 & 0.8719 & 3.0275 & 6.4060 & 0.3606 & 0.1371 \\
\hline 1 & 9 & 2 & 10 & 50 & 0.14420 & 0.1979 & 0.7285 & 8.0210 & 13.5763 & 1.6200 & 0.3168 \\
\hline 1.5 & 9 & 2 & 10 & 50 & 0.01390 & 0.0228 & 0.6085 & 9.7718 & 19.5763 & 3.7800 & 0.4968 \\
\hline 2 & 9 & 2 & 10 & 50 & 0.00054 & 0.0011 & 0.5082 & 9.9893 & 24.5880 & 6.8400 & 0.6768 \\
\hline 1.5 & 8 & 2 & 10 & 50 & 0.02230 & 0.0347 & 0.6430 & 9.6526 & 17.8497 & 3.3600 & 0.4416 \\
\hline 1.5 & 9 & 2 & 10 & 50 & 0.01390 & 0.0228 & 0.6085 & 9.7718 & 19.5763 & 3.7800 & 0.4968 \\
\hline 1.5 & 10 & 2 & 10 & 50 & 0.00863 & 0.0150 & 0.5758 & 9.8500 & 21.2101 & 4.2000 & 0.5520 \\
\hline 1.5 & 11 & 2 & 10 & 50 & 0.00536 & 0.0098 & 0.5449 & 9.9015 & 22.7563 & 4.6200 & 0.6072 \\
\hline 1.5 & 9 & 1.25 & 10 & 50 & 0.12510 & 0.1597 & 0.7837 & 8.4033 & 10.8150 & 1.8346 & 0.2437 \\
\hline 1.5 & 9 & 1.5 & 10 & 50 & 0.06650 & 0.0912 & 0.7284 & 9.0877 & 13.5841 & 2.3943 & 0.3169 \\
\hline 1.5 & 9 & 1.75 & 10 & 50 & 0.08340 & 0.1075 & 0.7756 & 8.9252 & 11.2205 & 2.2305 & 0.2541 \\
\hline 1.5 & 9 & 2 & 10 & 50 & 0.01390 & 0.0228 & 0.6085 & 9.7718 & 19.5763 & 3.7800 & 0.4968 \\
\hline 1.5 & 9 & 2 & 11 & 50 & 0.01910 & 0.0315 & 0.6065 & 10.6538 & 19.6757 & 3.4587 & 0.5001 \\
\hline 1.5 & 9 & 2 & 12 & 50 & 0.02500 & 0.0413 & 0.6048 & 11.5047 & 19.7583 & 3.1875 & 0.5028 \\
\hline 1.5 & 9 & 2 & 13 & 50 & 0.03140 & 0.0520 & 0.6034 & 12.3234 & 19.8280 & 2.9556 & 0.5051 \\
\hline 1.5 & 9 & 2 & 14 & 50 & 0.03830 & 0.0636 & 0.6022 & 13.1096 & 19.8876 & 2.7551 & 0.5071 \\
\hline 1.5 & 9 & 2 & 12 & 60 & 0.02710 & 0.0413 & 0.6570 & 11.5047 & 20.5772 & 3.1875 & 0.4200 \\
\hline 1.5 & 9 & 2 & 12 & 70 & 0.02880 & 0.0413 & 0.6972 & 11.5047 & 21.1925 & 3.1875 & 0.3606 \\
\hline 1.5 & 9 & 2 & 12 & 80 & 0.03010 & 0.0413 & 0.7291 & 11.5047 & 21.6716 & 3.1875 & 0.3159 \\
\hline 1.5 & 9 & 2 & 12 & 90 & 0.03120 & 0.0413 & 0.7549 & 11.5047 & 22.0550 & 3.1875 & 0.2811 \\
\hline
\end{tabular}

*=Seconds, $\$=$ Multiples of 10,000 packets/sec, 


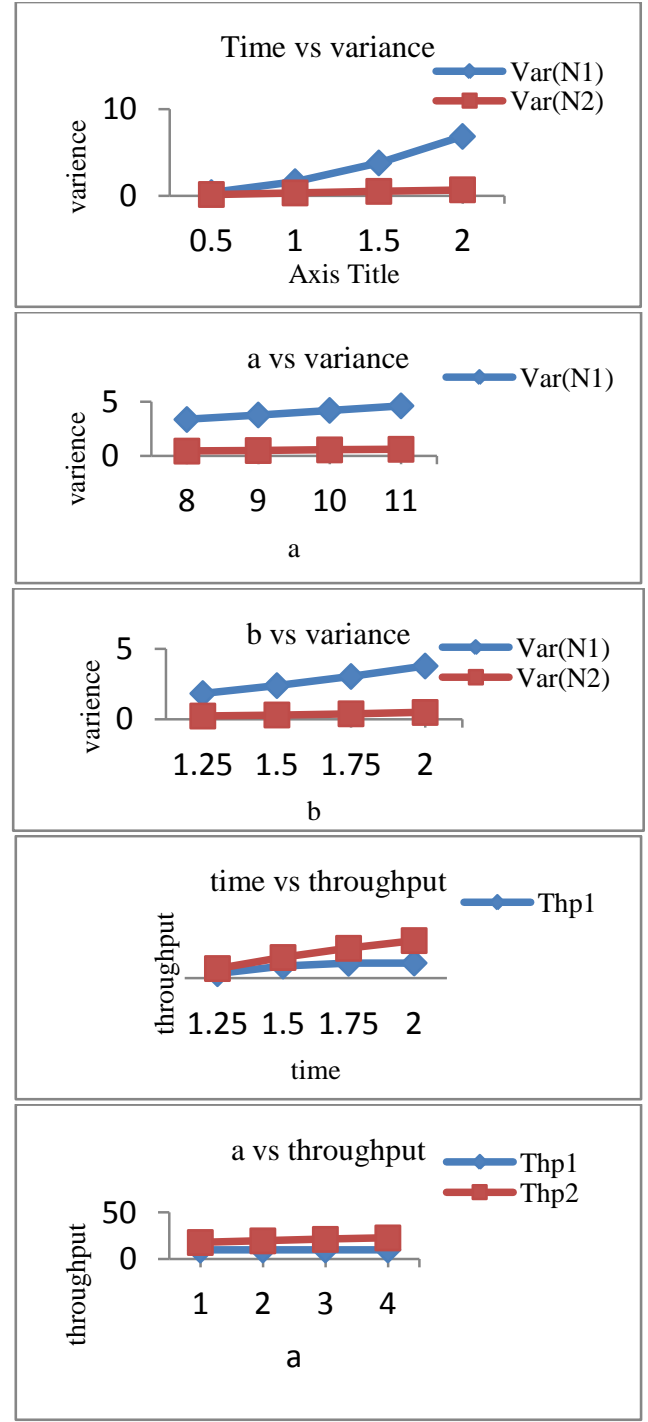

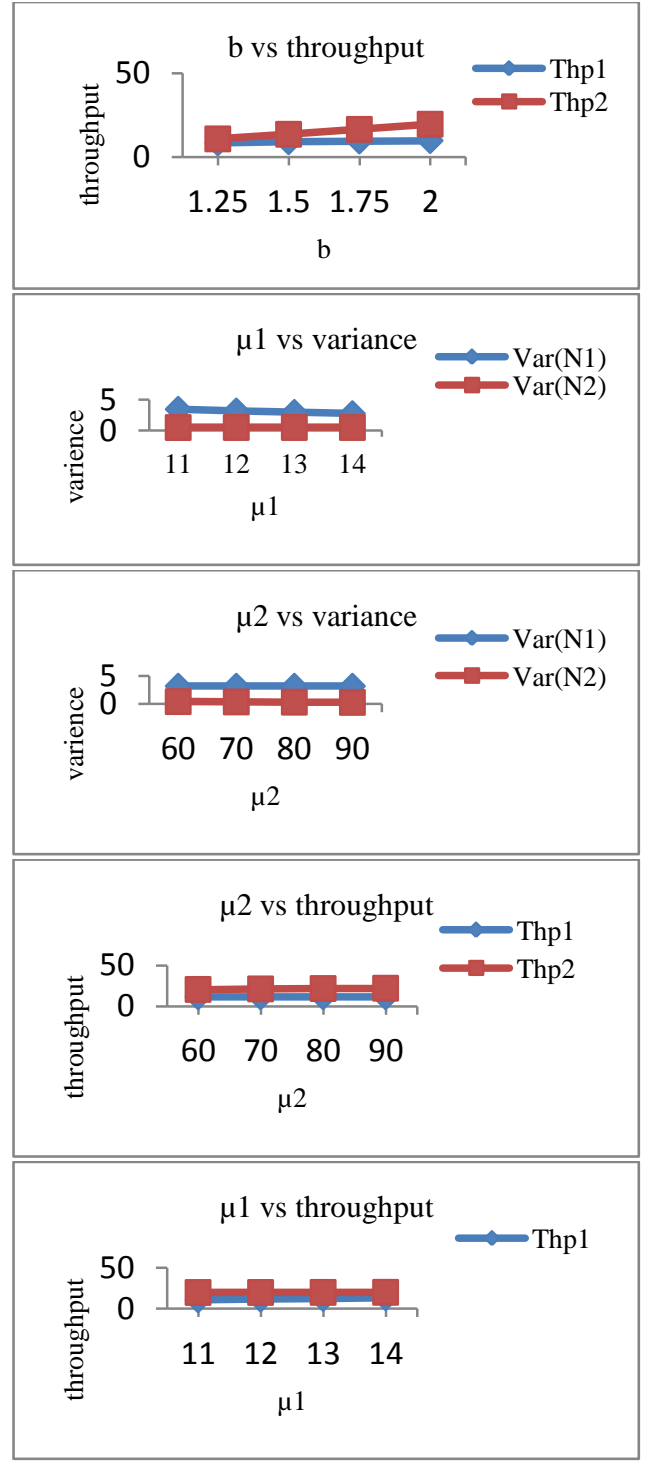

Figure 4: Relationship between throughput, variance and various parameters

It is observed as, time ' $t$ ' increases, the throughput of first and second nodes are increasing from 30275 packets to 99893 packets and from 6406 packets to 24588 packets respectively for fixed values of the other parameters. As the arrival rate parameter (a) varies from $8 \times 10^{4}$ packets/sec to $11 \times 10^{4}$ packets/sec, the throughput of the first buffer and the second buffer are increasing form 96526 packets to 99015 packets, 178497 packets to 227563 packets respectively, when other parameters remain fixed. The arrival rate parameter (b) varies from 1.25 to 2, the throughput of the first buffer and the second buffer are increasing from 84033 packets to 97718 packets, 108150 packets to 195763 packets respectively when the other parameters remain fixed.When the transmission rate $\mu_{1}$ varies from $11 \times 10^{4}$ packets/sec to $14 \times 10^{4}$ packets/sec, the throughput of the first buffer and the second buffer are increasing from 106538 packets to 131096 packets, 196757 packets to 198876 packets respectively when other parameters remain fixed. Similarly, the transmission rate $\mu_{2}$ varies from $60 \times 10^{4}$ packets/sec to $90 \times 10^{4}$ packets/sec, the throughput of the first buffer remains at 115047 packets and the throughput of the second buffer increases from 205772 packets to
22055 respectively when the other parameters remain fixed. If the variance of the number of packets in each buffer increases then the burstness of the buffers will be high. Hence, the parameters are to be adjusted in such a way that the variance of the content of each buffer is becomes small. The coefficients of variation of the buffer sizes are computed for each buffer which will help us to understand the consistency of the traffic flow through buffers. If this coefficient of variation is large then the flow is inconsistent and the requirement to search the assignable causes of high variation. It also helps us to control the smooth flow of packets in nodes. It is, also observed that the dynamic bandwidth allocation strategy has a significant influence on all performance measures of the network. It is further observed that the performance measures are highly sensitive towards smaller values of time. Hence, it is optimal to consider dynamic bandwidth allocation under and non-homogenous arrivals and evaluate the performance under transient conditions. It is observed that the congestion in buffers and delays in transmission can be reduced to a minimum level by adopting dynamic bandwidth allocation. This phenomenon has a vital bearing on quality of transmission. 


\section{SENSITIVITY ANALYSIS}

The following data has been considered for the sensitivity analysis:

$\mathrm{t}=1 \mathrm{sec}, \mathrm{a}=9 \times 10^{4}$ packets $/ \mathrm{sec}, \mathrm{b}=2, \mu_{1}=13 \times 10^{4}$ packets $/ \mathrm{sec}, \mu_{2}=60 \times 10^{4}$ packets $/ \mathrm{sec}$.

The sensitivity analysis for the mean number of packets, the utilization of nodes, the mean delay, and the throughput of the first and second buffers are computed and presented in the "Table 4" with variation of - $15 \%$,$10 \%,-5 \%, 0 \%,+5 \%,+10 \%$ and $+15 \%$ on the model parameters. The performance measures are highly affected by time (t) and arrival rate parameter (a). As $t$ increases from $-15 \%$ to $+15 \%$ the average number of packets in the two buffers and the total network increase along with the average delay in buffers, the utilization and the throughput of the two nodes. As arrival rate parameter (a) increases to $+15 \%$ the number of packets in the two buffers and total network are increasing along with the average delay, the utilization and the throughput of the two nodes. Similarly, for the arrival rate parameter (b), the utilization and the throughput are increasing in the communication network. Overall analysis of the parameters reflects that the dynamic bandwidth allocation strategy for congestion control will tremendously reduce the delay in communication and improve the voice quality by reducing burstiness in buffers.

Table.4 : Sensitivity Analysis of the communication network with Dynamic Bandwidth Allocation and Bulk Arrivals

\begin{tabular}{|c|c|c|c|c|c|c|c|c|}
\hline \multirow{2}{*}{$\begin{array}{l}\text { Parameter } \\
\text { time(t) sec }\end{array}$} & \multirow{2}{*}{$\begin{array}{c}\text { Performance } \\
\text { measure }\end{array}$} & \multicolumn{7}{|c|}{$\%$ change in parameters } \\
\hline & & $-15 \%$ & $-10 \%$ & $-5 \%$ & $0 \%$ & $5 \%$ & $10 \%$ & $15 \%$ \\
\hline \multirow{8}{*}{$\mathrm{t}=1$} & L1 & 0.9099 & 1.0257 & 1.148 & 1.278 & 1.4147 & 1.5582 & 1.7087 \\
\hline & L2 & 0.2269 & 0.2419 & 0.2569 & 0.2719 & 0.2869 & 0.3019 & 0.3169 \\
\hline & U1 & 0.5974 & 0.6414 & 0.6829 & 0.7214 & 0.757 & 0.7895 & 0.8189 \\
\hline & $\mathrm{U} 2$ & 0.203 & 0.2149 & 0.2266 & 0.2381 & 0.2494 & 0.2606 & 0.2716 \\
\hline & Thp1 & 7.7664 & 8.3388 & 8.8773 & 9.3787 & 9.841 & $\begin{array}{c}10.263 \\
4\end{array}$ & 10.6456 \\
\hline & Thp2 & 12.1811 & 12.893 & 13.5943 & 14.2852 & 14.9658 & $\begin{array}{c}15.636 \\
3\end{array}$ & 16.2968 \\
\hline & W1 & 0.1172 & 0.123 & 0.1294 & 0.1363 & 0.1438 & 0.1518 & 0.1605 \\
\hline & W2 & 0.0186 & 0.0188 & 0.0189 & 0.0190 & 0.0192 & 0.0193 & 0.0194 \\
\hline \multirow{8}{*}{$a=9$} & L1 & 1.0864 & 1.1503 & 1.2142 & 1.278 & 1.342 & 1.4059 & 1.4698 \\
\hline & L2 & 0.2311 & 0.2447 & 0.2583 & 0.2719 & 0.2855 & 0.2991 & 0.3127 \\
\hline & U1 & 0.6626 & 0.6835 & 0.7031 & 0.7214 & 0.7387 & 0.7549 & 0.7700 \\
\hline & $\mathrm{U} 2$ & 0.2064 & 0.2171 & 0.2277 & 0.2381 & 0.2484 & 0.2585 & 0.2685 \\
\hline & Thp1 & 8.6134 & 8.8849 & 9.1397 & 9.3787 & 9.6028 & 9.8132 & 10.0104 \\
\hline & Thp2 & 12.382 & 13.0251 & 13.6594 & 14.2852 & 14.9026 & $\begin{array}{c}15.511 \\
6\end{array}$ & 16.1124 \\
\hline & W1 & 0.1261 & 0.1295 & 0.1328 & 0.1363 & 0.1398 & 0.1433 & 0.1668 \\
\hline & W2 & 0.0187 & 0.0188 & 0.0189 & 0.0190 & 0.0192 & 0.0193 & 0.0194 \\
\hline \multirow{8}{*}{$\mathrm{b}=2$} & L1 & 1.1119 & 1.1682 & 1.2235 & 1.278 & 1.3319 & 1.3848 & 1.4370 \\
\hline & L2 & 0.2378 & 0.2494 & 0.2608 & 0.2719 & 0.2829 & 0.2936 & 0.3041 \\
\hline & U1 & 0.6711 & 0.6891 & 0.7058 & 0.7214 & 0.736 & 0.7496 & 0.7624 \\
\hline & $\mathrm{U} 2$ & 0.2117 & 0.2208 & 0.2296 & 0.2381 & 0.2464 & 0.2544 & 0.2611 \\
\hline & Thp1 & 8.7239 & 8.9578 & 9.1756 & 9.3787 & 9.5682 & 9.7452 & 9.9106 \\
\hline & Thp2 & 12.7004 & 13.2451 & 13.7731 & 14.2852 & 14.7821 & $\begin{array}{c}15.264 \\
4\end{array}$ & 15.7326 \\
\hline & W1 & 0.1257 & 0.1304 & 0.1333 & 0.1363 & 0.1392 & 0.1421 & 0.1450 \\
\hline & W2 & 0.0187 & 0.0188 & 0.0189 & 0.0190 & 0.0191 & 0.0192 & 0.0193 \\
\hline \multirow{8}{*}{$\mu_{1}=13$} & L1 & 1.4815 & 1.407 & 1.3395 & 1.278 & 1.2221 & 1.1707 & 1.1235 \\
\hline & L2 & 0.2679 & 0.2694 & 0.2707 & 0.2719 & 0.273 & 0.2740 & 0.2749 \\
\hline & U1 & 0.7727 & 0.7551 & 0.738 & 0.7214 & 0.7054 & 0.6899 & 0.6749 \\
\hline & $\mathrm{U} 2$ & 0.235 & 0.2361 & 0.2372 & 0.2381 & 0.2389 & 0.2397 & 0.2404 \\
\hline & Thp1 & 8.5385 & 8.8349 & 9.1145 & 9.3787 & 9.6285 & 0.8649 & 10.089 \\
\hline & Thp2 & 14.0987 & 14.1679 & 14.2297 & 14.2852 & 14.3354 & 14.381 & 14.4226 \\
\hline & W1 & 0.1735 & 0.1593 & 0.147 & 0.1363 & 0.1264 & 0.1187 & 0.1114 \\
\hline & W2 & 0.0190 & 0.0190 & 0.0190 & 0.0190 & 0.0190 & 0.0190 & 0.0190 \\
\hline \multirow{8}{*}{$\mu_{2}=60$} & L1 & 1.2781 & 1.2781 & 1.2781 & 1.2781 & 1.2781 & 1.2781 & 1.2781 \\
\hline & L2 & 0.3189 & 0.3015 & 0.2860 & 0.2719 & 0.2592 & 0.2476 & 0.2370 \\
\hline & U1 & 0.7214 & 0.7214 & 0.7214 & 0.7214 & 0.7214 & 0.7214 & 0.7214 \\
\hline & $\mathrm{U} 2$ & 0.2730 & 0.2603 & 0.2487 & 0.2381 & 0.2283 & 0.2193 & 0.2110 \\
\hline & Thp1 & 9.3787 & 9.3787 & 9.3787 & 9.3787 & 9.3787 & 9.3787 & 9.3787 \\
\hline & Thp2 & 13.9246 & 14.0566 & 14.1762 & 14.2852 & 14.3849 & $\begin{array}{c}14.476 \\
5\end{array}$ & 14.5608 \\
\hline & W1 & 0.1363 & 0.1363 & 0.1363 & 0.1363 & 0.1363 & 0.1363 & 0.1363 \\
\hline & W2 & 0.0229 & 0.0215 & 0.0202 & 0.0190 & 0.0180 & 0.0171 & 0.0163 \\
\hline
\end{tabular}




\section{COMPARATIVE STUDY}

The comparative study between the proposed communication network models with homogenous Poisson arrivals it's carried in this section. The computed performance measure of both models are presented in the "Table 5" for different values of $\mathrm{t}=1,1.5,2,2.5$ seconds.As $\mathrm{t}$ increases, the percentage variation of performance measures between the models also increases.
For the proposed model with non-homogenous arrivals having dynamic bandwidth allocation has more utilization compared to that of the model with Poisson arrivals under dynamic bandwidth allocation. It is, also observed that the assumption of non-homogenous arrivals has a significant influence on all the performance measures of the network.

Table 5: Comparative study of models with Homogeneous and Non-Homogeneous Duane arrivals

\begin{tabular}{|c|c|c|c|c|c|}
\hline $\begin{array}{c}\text { Time(t) } \\
\text { sec }\end{array}$ & $\begin{array}{c}\text { Parameters } \\
\text { measured }\end{array}$ & $\begin{array}{c}\text { Model with } \\
\text { Poisson arrivals }\end{array}$ & $\begin{array}{c}\text { Proposed } \\
\text { Model }\end{array}$ & Difference & $\%$ of Variation \\
\hline \multirow{8}{*}{$\mathrm{t}=1$} & L1 & 0.7500 & 1.375 & 0.6250 & 45.45454545 \\
\hline & $\mathrm{L} 2$ & 0.5000 & 0.8611 & 0.3611 & 41.93473464 \\
\hline & U1 & 0.5276 & 0.7472 & 0.2196 & 29.38972163 \\
\hline & $\mathrm{U} 2$ & 0.3935 & 0.5773 & 0.1838 & 31.83786593 \\
\hline & Thp1 & 6.3316 & 8.9659 & 2.6343 & 29.38132257 \\
\hline & Thp2 & 0.0823 & 10.3916 & 10.3093 & 99.20801417 \\
\hline & W1 & 0.1185 & 0.1534 & 0.0349 & 22.75097784 \\
\hline & W2 & 0.0706 & 0.0829 & 0.0123 & 14.8371532 \\
\hline \multirow{8}{*}{$\mathrm{t}=1.5$} & L1 & 1.1250 & 3.1875 & 2.0625 & 64.70588235 \\
\hline & L2 & 0.5000 & 1.3611 & 0.8611 & 63.26500624 \\
\hline & U1 & 0.6753 & 0.9587 & 0.2834 & 29.56086367 \\
\hline & $\mathrm{U} 2$ & 0.3935 & 0.7436 & 0.3501 & 47.08176439 \\
\hline & Thp1 & 8.1042 & 11.5047 & 3.4005 & 29.5574852 \\
\hline & Thp2 & 7.0824 & 13.3852 & 6.3028 & 47.08782835 \\
\hline & W1 & 0.1388 & 0.2771 & 0.1383 & 49.90977986 \\
\hline & W2 & 0.0706 & 0.1017 & 0.0311 & 30.58013766 \\
\hline \multirow{8}{*}{$t=2$} & L1 & 1.5000 & 5.7500 & 4.2500 & 73.91304348 \\
\hline & $\mathrm{L} 2$ & 0.5000 & 1.8611 & 1.3611 & 73.13416797 \\
\hline & U1 & 0.7769 & 0.9968 & 0.2199 & 22.0605939 \\
\hline & $\mathrm{U} 2$ & 0.3935 & 0.8445 & 0.451 & 53.40438129 \\
\hline & Thp1 & 9.3224 & 11.9618 & 2.6394 & 22.06524102 \\
\hline & Thp2 & 7.0824 & 15.201 & 8.1186 & 53.4083284 \\
\hline & W1 & 0.1609 & 0.4807 & 0.3198 & 66.52798003 \\
\hline & W2 & 0.0706 & 0.1224 & 0.0518 & 42.32026144 \\
\hline \multirow{8}{*}{$\mathrm{t}=2.5$} & L1 & 1.8750 & 9.0625 & 7.1875 & 79.31034483 \\
\hline & L2 & 0.5000 & 2.3611 & 1.8611 & 78.82342976 \\
\hline & U1 & 0.8466 & 0.9999 & 0.1533 & 15.33153315 \\
\hline & $\mathrm{U} 2$ & 0.3935 & 0.9047 & 0.5112 & 56.50491876 \\
\hline & Thp1 & 10.1597 & 11.9986 & 1.8389 & 15.32595469 \\
\hline & Thp2 & 7.0824 & 16.3023 & 9.2199 & 56.55582341 \\
\hline & W1 & 0.1846 & 0.7553 & 0.5707 & 75.55938038 \\
\hline & W2 & 0.0706 & 0.1448 & 0.0742 & 51.24309392 \\
\hline
\end{tabular}

\section{CONCLUSION}

In this paper we have introduced a new and novel two node tandem communication network model with Duane arrival process having dynamic bandwidth allocation. The arrival of packets in a network traffic is characterized through Weibull inter arrival time distribution which better represents the self-similarity network traffic having time dependent burstness. The Duane process is a generalization of Poisson process. Here it is, assumed that the two nodes are connected in tandem and the transmission processes follow Poisson. The transmission rate at every instant of packet transmission is adjusted depending on the content of the buffer connected to it. The explicit expressions for the performance measures of the communication network such as mean content of the buffers mean delays in transmission, the throughput of the nodes, utilization of the buffers are derived. The sensitivity of the model revealed that the inter arrival rate parameters has significant influence on predicting the performance measures of the network more accurately. A comparative study of the proposed network with that of inter exponential transmission times shown that the proposed model outperform the existing communication network 
model in scheduling self-similarity networks such as LAN, WAN and MAN. It is, also possible to derive the optimal operating policies of the networks with suitable cost consideration which will be taken elsewhere.

\section{REFERENCES}

[1] V. Frost and B.Melarned, (1994) "Traffic modeling for telecommunication networks," IEEE commun. Magazine., vol.33, pp. 70-80.

[2] P.Abry, R. Baraniuk, P.Flandrin, R. Riedi, D. Veitch, (2002) "Multi-scale Nature of Network Traffic," IEEE Signal Processing Magazine, pp. 28-27.

[3] Cappe O, E. Moulines, J. C. Pesquet, A.Petropulu, X Yang (2002), "Long-Range Dependence and HeavyTail Modeling for Teletraffic Data," IEEE signal Processing Magazine, pp.14-27.

[4] P. Danzig, S.Jamin, R. Caceres, D.Mitzel, and D. Estrin, (1992) "An empirical workload model for driving wide-area TCP/IP network simulations," Internetworking: Res., Experience, vol. 3, no. 1, pp. $1-26$.

[5] H. Fowler and W. Leland, (1991), "Local area network traffic characteristics, with implications for broadband network congestion management," IEEE J. Select areas commun, vol.9. pp. 1139-1149.

[6] R. Gusella (1990), “A measurement study of diskless workstation traffic on an Ethernet, "IEEE Trans. Commun., vol. 38, pp. 1557-1568.

[7] R.Jain and S.Routhier, (1986), "Packet trainsMeasurements and a new model for computer

[8] network traffic, "IEEE J. Select. Areas Commun., Vol. 4, pp. 986-995.

[9] W.Leland, M. Taqqu, W. Willinger, and D. Wilson,(1994) "On the self-similar nature of Ethernet traffic(extended version)," IEEE/ACM Trans. Networking, vol. 2, pp. 1-15.

[10] V. Paxson and S. Floyd, (1995), "Wide area traffic: the failure of Poisson modeling," IEEE/ACM Trans. Netw., vol.3, no.3, pp. 226-244.

[11] M. V. Rama Sundari, K. Srinivasa Rao, P. Srinivasa Rao, P. Suresh Varma, (2011), On Tandem Communication Network Model with DBA having NHP Arrivals for First Node and Poisson process arrivals for Second Node in International Journal of Computer Science $(136-144)$ Volume 8 , No. 2.
[12] M. V. Rama Sundari, K. Srinivasa Rao, P. Srinivasa Rao, P. Suresh Varma, (2011), Three Node Tandem Communication Network Model with Dynamic Bandwidth Allocation and Non Homogeneous Poisson Arrivals in International Journal of Computer Applications (0975 - 8887) Volume 31, No. 1

[13] Trinatha Rao, P., K.Srinivasa Rao, (2012) ,Performance of non-homogeneous communication with Poisson arrivals and dynamic bandwidth allocation, International Journals of Systems, control and communication, Vol. 4 No. 3, pp 164-182.

[14] Suhasini, A.V.S, P.srnivasa Rao, P.R.S.Reddy (2013a), Transient analysis of tandem queuing model with non-homogeneous Poisson bulk arrivals having state dependent service rates, International Journal of Advanced Computer and mathematical Sciences, Vol. 3,No. 3, pp 272-284.

[15] Suhasini, A.V.S, P.srnivasa Rao, P.R.S.Reddy (2013), Transient analysis of parallel and series queuing model with non-homogeneous Poisson binomial arrivals having independent service rates, Normal parallel and scientific computations, Vol.21,No.2, pp 235-262.

[16] Suhasini, A.V.S., K.Srnvasa Rao, P.R.S.Reddy (2013b), on parallel and series non-homogeneous bulk arrival queuing model, OPSEARCH, Vol. 50, No. 4, pp 521-547.

[17] Rakesh Singhai, Shiva Dutt Joshi and Rajendra K. P. Bhatt, (2007), "A Novel Discrete Distribution and Process to Model Self-Similar Traffic, "9 $9^{\text {th }}$ IEEE international conference on telecommunications Con TEL 2007, Zagreb, Croatia, pp 167-171.

[18] Earlang, A.K.(1909). Probablity and Telephone calls, Nyt.Tidsskr Krarup Mat. Ser. B. vol.20,pp 33-39.

[19] A. Feldmann, (2000), "characteristics of TCP connection Arrivals" chapter 15, Self-Similar Network traffic and Performance evolution, edited by K.Park, W. Willinger,jhon wiley \& Sons Inc.

[20] M. J. Fisher, D. Gross, D. Masi and J. F. Shortle, (2001), "Analyzing the Waiting Time Process in Internet Queuing Systems With the Transform Approximation Method," The Telecommunications Review,12, pp 21-32. 